\title{
Electrical Energy Harvesting From Mechanical Pressure of Vehicles Using Piezoelectric Generators
}

\author{
Ashish Dakhole ${ }^{1}$, Amol Boke ${ }^{2}$ \\ ${ }^{1}$ (B.E. Student, ECE Dept., GHRAET, Nagpur, India) \\ ${ }^{2}$ (Asst, Prof., ECE Dept, GHRAET, Nagpur, India)
}

\begin{abstract}
The paper is intended to forecast the present condition of energy utilization and the usage of the resources in the generation process. According to International Energy Agency's New Policies Scenario demand of global electricity consumption will grow by almost $80 \%$ by 2040.The contribution of renewable resources in accordance with power generation will increase up to 33\% by 2040. The rapidly-increasing contribution and importance of renewable resources in power generation field has led to developments of several sustainable technologies. It's an opportunity to generate a clean and green energy using the resources what we have in present. Piezoelectricity is a very old discovery not much been developed yet and not much trusted due to its inefficiency. The recent research and development in the electronics sector and its applied streams of electricity production and storage techniques have made possible to capture the energy that was always unnoticed so far. Energy which is in fact invisible energy which can be generated by applying pressure on specific substances with varying pressure produced by the on road moving vehicles. Thus, making the Piezoelectric effect a sensible concept. It is a new concept and has greater prospects in the maintainable energy circles. It is relatively inexpensive and easy to install, and recycles otherwise wasteful forms of energy. Here we mention that the conditions presented are approximations and recent practices have shown that greater amount of energy can be generated from such practices.
\end{abstract}

Keywords: Energy harvesting, piezoelectricity, eco-friendly, green energy, piezoelectric generators, Vibrational Energy.

\section{Introduction}

In India generally $18-20 \%$ of the total energy bill goes towards street lighting and therefore this is one domain that needs major attention.Our objective is to harvest mechanical pressure generated from running vehicles on road into electrical energy and store the generated power for further use. The concept of piezoelectricity to generate electricity form the moving vehicles on road is being used. There is a lot of variations in traffic density, much traffic during the day time than night. By embedding the piezoelectric generators in the road can convert the pressure created by vehicles into sensible form of electricity. Presently, most commercial ones are the asphalt roads on which most of the vehicles runs. From the assumptions it is clear that greater amount of electrical energy can be generated from normal vehicles like cars, bikes etc. Also it has the ability to survive the load created by the heavy vehicles having tones of load transforming into a much greater amount of electric power [1]. The generated power is stored in the batteries and can be used further for roadside applications e.g. street lights, advertisement boards.

\section{Piezoelectric Effect}

Piezoelectric effect is the capability of certain substances to generate an electric potential in response to applied mechanical stress. The term piezoelectricity means electricity generated by applying pressure. The term is derived from Greek word piezein, means to press, and elektron, an ancient source of electric charge. It was discovered by French physicist Jacques and Pierre Curie.

Piezoelectricity is the relation between mechanical stress (pressure) and electrical voltage. On applying force on the piezoelectric material, results in the production of charge in the material. When no force is applied, the piezoelectric substance is in balance condition and the positive and negative charges are evenly distributed. On application of force over the material it slightly deformed disturbing the lattice structure of the material resulting in the generation of electric potential. The electrical charge is accumulated over the crystal surface that can be extracted using wires [2].

There are two types of effects in piezoelectricity: direct piezoelectric effect and converse piezoelectric effect. The direct piezoelectric effect is related to the internal generation of electric charge resulting from applied mechanical force whereas; the converse effect is related to the internal generation of mechanical stress resulting from applied electric potential. The converse effect is used in the production of piezoelectric actuators. 


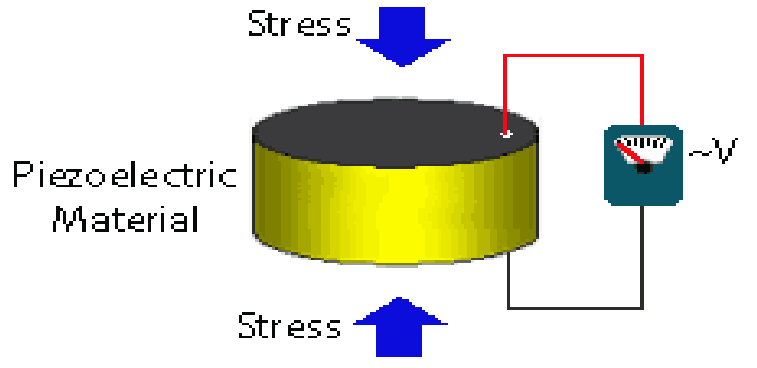

Piezoelectric Effect

Fig. 1 Graphical representation of the mechanism of the direct piezoelectric effect.

\section{Methodology}

In today's society motor vehicles are one of the most necessary mean of transportation and it is convenient and flexible. There are significant number vehicles on road generating million joules of mechanical energy which is been wasted. There are a lot of variations in traffic density, much traffic during the day time than night on roads and highways. By embedding the piezoelectric generators in the road which will convert the pressure created by vehicles into sensible form of electricity. Presently, most commercial ones are the asphalt roads on which most of the vehicles runs. Also it has the ability to survive the load created by the heavy vehicles having tones of load transforming into a much greater amount of electric power. The generated power is stored in the batteries and can be used further for roadside applications e.g. street lights, advertisement boards.

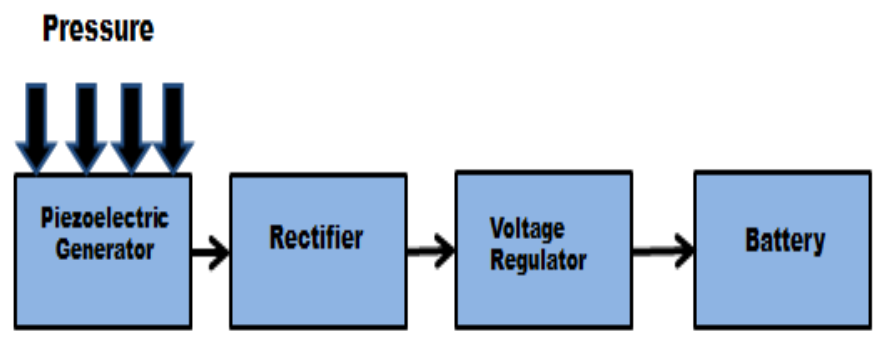

Fig. 2 Piezoelectric Energy Harvesting Process.

The block diagram shows the process involved in the harvesting process. Piezoelectric generator is a type of device which works on the principle of piezoelectric effect. The piezoelectric generator is used to convert the mechanical pressure exerted by the vehicle on road to electrical energy. The output of the generator is an AC voltage. So to store the charge into batteries it has to be first converted into DC voltage. Rectifier is a circuit which is used to convert the AC into equivalent DC. Here full-wave bridge rectifier is used. The bridge rectifier is converting the incoming $\mathrm{AC}$ into equivalent $\mathrm{DC}$ voltage. The voltage source in a circuit may have fluctuations and would not give the fixed voltage output therefore, voltage regulator is used. The voltage regulator maintains the output voltage at a constant value. The final output is given to the battery charging circuit.

\section{Hardware Setup}

To show electricity generation by method mentioned above in this paper a demonstrative hardware setup is being build. In this 2 wooden plates (Dimension) are taken and piezoelectric generators are embedded in-between these 2 wooden plates. These piezoelectric generators will generate the electrical energy upon exerting mechanical pressure. This electricity is measured by multi-meter in millivolts. Now this generated electrical energy is given to rectifier circuit to convert fluctuating electrical energy into steady DC output. This DC form of electricity will be stored in battery by using coupled capacitors.

As shown in pictures below after applying the pressure by simple human hand, setup is generating electrical signals which are shown by multi-meter. The pressure can be applied by a hand, a foot or a vehicle but it has to be instantaneous i.e. electricity will not keep on generating 5 seconds after applying pressure. Electricity generated by this setup as soon as pressure is applies to pressure plates. 

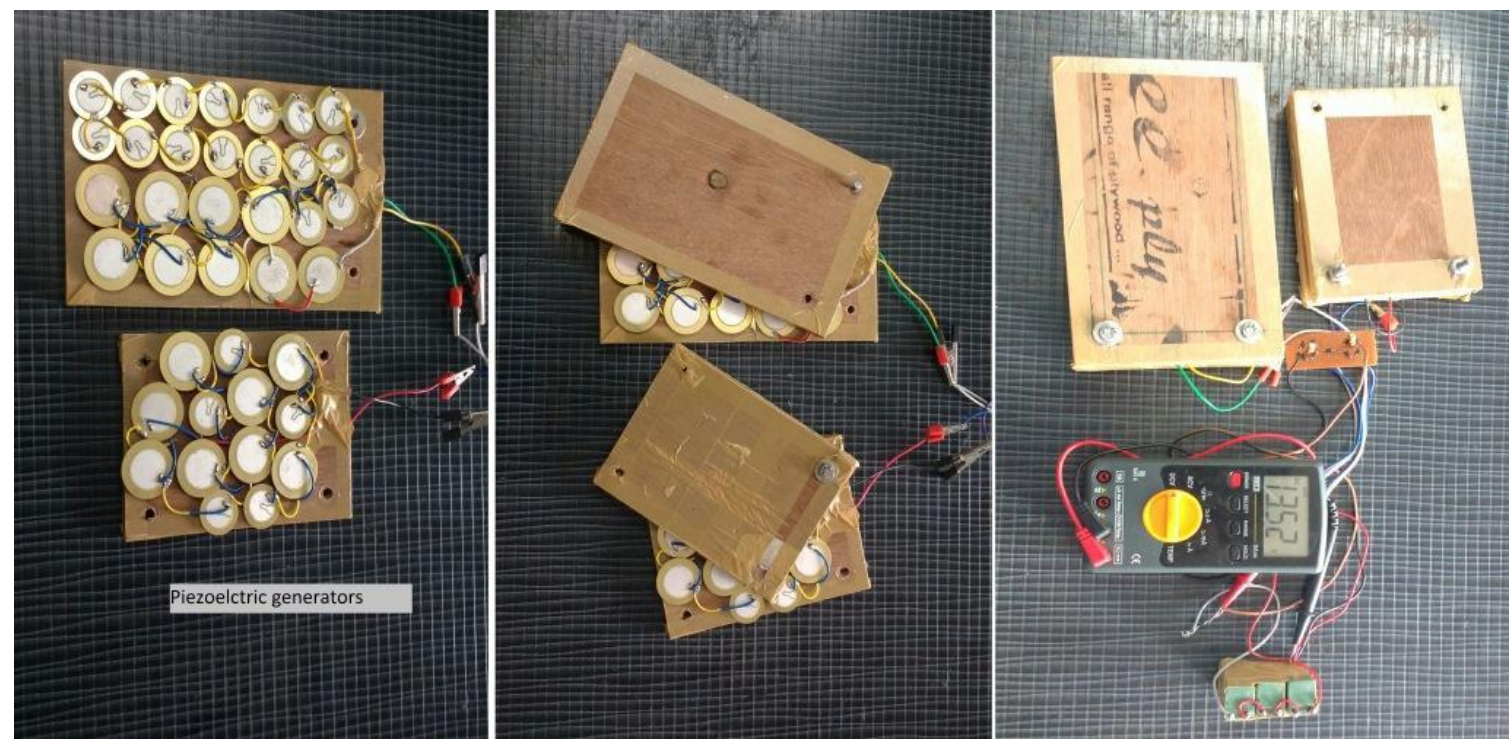

Fig. 3 Demonstrative Hardware setup with Piezoelectric generators.

\section{Efficient Scenario For Large Scale Generation Of Electrical Power From Piezoelecticity}

Idea of embedding the piezoelectric generators in the roads to convert the wasted mechanical pressure by vehicles into useful form of electrical energy in being incorporated in this paper. As nowadays the most commercial roads are asphalt roads (Tar roads) consisting of several layers on which several vehicles run. The first layer laid consists of fine gravels and sand content. Then a thin layer of asphalt is laid which acts like a strong base for the generators. Then the piezoelectric generators are placed in quick drying concrete. Then all the generators are wired in series to get the collective output of all generators. A bitumen sheet is used to cover all the generators to provide better adhesion between concrete and asphalt. At last a thick layer of asphalt is laid which completes the construction.

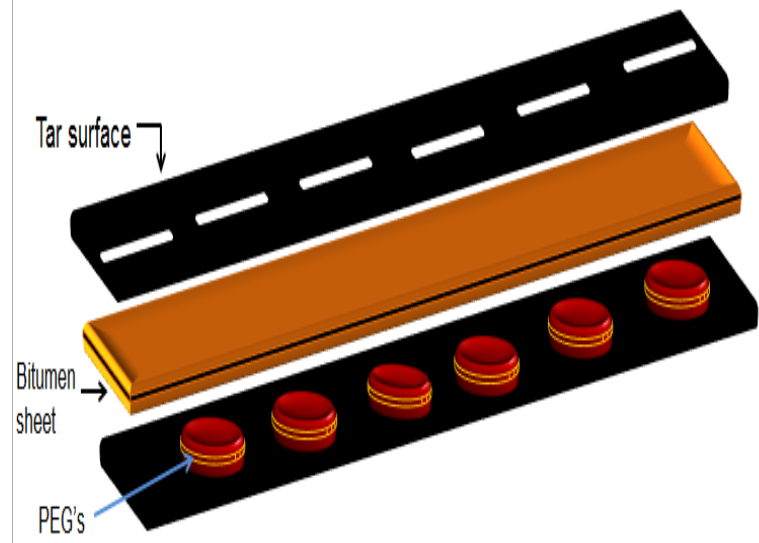

Fig.4 Schematic of PEG's embedded roads.

Though the dimensions of the generator are not fixed it can be designed accordingly. Recent experimentation carried by Innowattech in Israel, consists of putting PEG's (Piezoelectric Electric Generators) $6 \mathrm{~cm}$ under the road level and at a distance of $30 \mathrm{~cm}$ apart, and the whole system of being covered by layer asphalt. From trials, it has been observed that a tuck whose weight is around 5tons can generate about 2000V, and $1 \mathrm{~km}$ stretch of generators along a dual carriageway assuming 600 vehicles go through that road segment in 1hour, can generate about 200kWh much enough energy.

Similar method if we try to implement. Based on our calculations, we are considering an area about 100 sq.ft $\&$ generation of power by single compact car.

\section{Supplied Data:-}

Generator Size=1sq.ft.

Required no. of generators $=49$.

Cost of 1 generator $=$ Rs.2000 approx.

(Distance between two consecutive generator $=0.5 \mathrm{ft}$ ) 
Weight of a compact car $=1354 \mathrm{~kg}$.

Total charge generated $=1354 * 0.4=540 \mathrm{~V}$ approx.

The minimum lifetime of this piezoelectric road is 15 years and also from the calculation we can observe that sensible amount of power is been generated making benefits of the invested money in much smarter way. The most profitable sides of this project is the green solution for generating power, conserving the energy used in road applications, working in all weather conditions and continuous generation. It doesn't require any additional space and easily feasible to be fitted in current system.

\section{Applications}

As the structure of the proposed system is compact, therefore it can be fitted in any structure increasing the areas of the applications. It can also be installed on footpaths to capture the wasted energy by the movement of peoples. It can also be used in runways converting the massive amount of pressure exerted by the aircrafts while landing and take-off converting it into huge amount of electrical potential. Also the system can be fitted in railway tracks in order to convert the pressure applied by the trains on tracks to a useful amount of energy. Piezoelectricity makes productive use of the wasteful energy that remains untapped every single time. It also could make a good amount of energy from high traffic area such a station platforms, and the areas having large number of public.

By implementing this concept we can assure to save energy utilized for public utilities such as street light, Traffic signaling of vehicles as well as railways by making them work on this harvested electrical energy at least $20 \%$ of their functioning total time.

\section{Conclusion}

The recent venture on eco-friendly sources of energy has opened new doors for the global energy market. Piezoelectricity offers a valid alternative to conventional fuels. It is much cheaper and relatively easy to install and recycles the wasteful energy. However it should be mentioned that the scenario presented here is based on approximations and recent experimentation had proved that large amount of energy can be generated using the full amount of generated energy. Currently the efficiency is low, in range of about 20 to $30 \%$. More development is required in this field in order to increase its efficiency and to develop efficient methods of utilization. As the field of electronics is advancing day by day, better synthesized crystals, efficient generators and better palaces for installation generating more amount of electricity and can be viewed as the next promising source for generation of electricity

\section{Acknowledgements}

The authors would like to thank Department of Electronics \& Communication Engineering, G. H. Raisoni Academy of Engineering and Technology, Nagpur for their valuable support, advice and technical guidance during the presentation of this paper.

\section{Journal Papers:}

\section{References}

[1] Debayan Paul, Anupam Roy, Smart roads in green energy harvesting, International Journal of Engineering and Technical Research (IJETR)ISSN: 2321-0869, Volume-3, Issue-2, February 2015.

[2] Rupendra Kumar Gohite, Madhuri Gohite, Generation of electricity thorough PZT materials with the help footfall stress, International Journal Of Core Engineering \& Management (IJCEM) Volume 1, Issue 5, August 2014.

[3] Mukti Nath Gupta, Suman and S.K. Yadav Electricity Generation Due to Vibration of Moving Vehicles Using Piezoelectric Effect Advance in Electronic and Electric Engineering.ISSN 2231-1297, Volume 4, Number 3 (2014), pp. 313-318

[4] J. John Livingston and M. Hemalatha Charging an Electronic Gadget using Piezoelectricity Indian Journal of Science and Technology, Vol 7(7), 945-948, July 2014 